\title{
Competencias profesionales en Educación para la Sostenibilidad: un estudio exploratorio de la visión de futuros maestros
}

\section{Professional competencies in Education for Sustainability: an exploratory study of student teachers' views}

\author{
Gisela Cebrián \\ University of Southampton \\ G.Cebrian-Bernat@soton.ac.uk
}

\author{
Mercè Junyent Pubill \\ Departament de Didàctica de la Matemàtica i de les Ciències Experimentals, UAB \\ merce.junyent@uab.cat
}

\begin{abstract}
RESUMEN • La investigación que se presenta en este artículo se basa en un estudio exploratorio sobre la visión de un grupo de futuros maestros de educación primaria en relación a la Educación para la Sostenibilidad desde un punto de vista competencial. En primer lugar se redefine un marco conceptual de competencias profesionales de los maestros en Educación para la Sostenibilidad, basado en la especificación del concepto de competencia, el rol como formadores, las competencias generales y el desarrollo profesional en Educación para la Sostenibilidad. Este marco competencial a su vez orienta la exploración de la visión sobre competencias profesionales de los futuros maestros, que constituye el segundo objetivo de esta investigación. El instrumento utilizado es un cuestionario al que han respondido un grupo de 32 alumnos de 3..$^{\circ}$ de Magisterio de la diplomatura de Educación Primaria, el análisis del cual ha sido básicamente cualitativo. Algunos de los resultados indican que los futuros/as docentes, participantes en el estudio, enfatizan la clarificación de valores, la capacidad de tomar decisiones, participar y actuar para el cambio, pero por el contrario no consideran visionar escenarios de futuro, el pensamiento crítico, el diálogo entre disciplinas y la gestión de las emociones y preocupaciones, competencias profesionales esenciales en la Educación para la Sostenibilidad. Se considera que los resultados obtenidos pueden orientar el diseño de estrategias didácticas y herramientas de evaluación para la mejora de la formación inicial del profesorado en el ámbito de las competencias profesionales en Educación para la Sostenibilidad.
\end{abstract}

PALABRAS CLAVE: Educación para la Sostenibilidad; competencias profesionales; formación inicial del profesorado; educación superior; desarrollo profesional.

ABSTRACT - The research presented in this paper is based on an exploratory study on the views of a group of future primary education teachers in Education for Sustainability from a competence standpoint. First, we redefine a conceptual framework of professional competencies of teachers in Education for Sustainability. This framework is based on the competence conceptualisation, the role as trainer, generic competencies and professional development on Education for Sustainability. In turn this competencies framework guides the exploration of the student teachers' views, which corresponds to the second objective of this research. The research instrument used is a questionnaire. A group of thirty-two third-year students on the Bachelor of Primary Education responded to our questionnaire, the analysis of which was qualitative. The findings indicate that student teachers, the participants in this study, emphasise values clarification, decision-making, participation and action for change, however they discount envisioning future scenarios, critical thinking, interdisciplinary dialogue and the management of emotions, essential professional competencies in Education for Sustainability. The findings of this study can guide the design of educational strategies and assessment tools to improve teacher training in the field of professional competencies in Education for Sustainability.

KEYWORDS: Education for Sustainability; professional competencies; pre-service teacher training; higher education; professional development.

Fecha de recepción: febrero 2012 • Aceptado: setiembre 2012

Cebrián, G. y Junyent, M. (2014). Competencias profesionales en Educación para la Sostenibilidad: un estudio exploratorio de la visión de futuros maestros. Enseñanza de las Ciencias, 32 (1), pp. 29-49 


\section{INTRODUCCIÓN}

El nuevo modelo de educación dentro del Espacio Europeo de Educación Superior (EEES), iniciado en 1999 con la Declaración de Bolonia (Bolonia, 1999), basado en la enseńanza-aprendizaje por competencias propone algunos cambios importantes e interesantes y deja atrás un modelo centrado en el profesorado y la enseńanza, para adoptar un modelo centrado en el alumnado y en su aprendizaje, que promueve de manera activa el pensamiento crítico, la contextualización, la acción y la cohesión social. Desde nuestra perspectiva ello supone una oportunidad para introducir lo que la Educación para la Sostenibilidad (ES) viene reclamando desde hace unas décadas: una educación para una transformación social sostenible, que estimula la reflexión crítica y la clarificación de valores, promueve el pensamiento sistémico y es innovadora y constructiva, culturalmente apropiada y orientada hacia la acción (Huckle y Sterling, 1996; Tilbury y Wortman, 2004).

En este artículo se presenta una investigación en la que se ha redefinido un marco competencial para profesorado de Educación Primaria en Educación para la Sostenibilidad, a través de la triangulación de tres referentes teóricos en ES: CSCT Project (Sleurs, 2007), Modelo ACES (Junyent, Geli y Arbat, 2003) y Criterios de Calidad para Escuelas Sostenibles (Breiting, Mayer y Mogensen, 2005). Este marco orienta una investigación exploratoria sobre la visión de las competencias profesionales en ES en futuros docentes de Educación Primaria. La obtención de datos se ha realizado a través de un cuestionario al que han respondido 32 futuros maestros. Obtener la visión de los futuros maestros es relevante, por la cantidad de horas lectivas que impartirán y la capacidad que tendrán en clarificar valores y comportamientos en su alumnado, siendo necesaria su capacidad para introducir de manera transversal la ES en el currículum (UNESCO, 2009).

Este estudio pretende contribuir a la necesidad de llevar a cabo investigaciones que clarifiquen la definición de competencias del profesorado en ES y que puedan orientar trabajos posteriores sobre las estrategias educativas y los instrumentos evaluativos para una integración efectiva de la ES en la formación del profesorado.

\section{JUSTIFICACIÓN DE LA INVESTIGACIÓN, CONTEXTO Y OBJETIVOS}

Ante la creciente globalización la sociedad se enfrenta a nuevos retos donde los ciudadanos necesitan desarrollar un conjunto de competencias que les permitan ser flexibles y capaces de poder interactuar en un mundo situado bajo la influencia del cambio (Delors, 1996; CEE, 2006; OCDE, 2002). La sostenibilidad constituye uno de estos retos fundamentales de nuestra civilización y afrontarlo comporta, entre otros aspectos, dotar al conjunto de agentes educativos de herramientas conceptuales y metodológicas que faciliten la presencia de la Educación para la Sostenibilidad (ES) en diversidad de contextos educativos. En este sentido, en el año 2002, durante la Segunda Cumbre de la Tierra en Johannesburgo, Naciones Unidas declaró la Década de Educación para el Desarrollo Sostenible (2004-2015), en la que se pone en evidencia un consenso a nivel internacional por el que se considera que el avance hacia el desarrollo sostenible ${ }^{1}$ supone la necesidad de establecer marcos de actuación que faciliten la participación, la concienciación, la educación y la capacitación de la ciudadanía (UNESCO, 2004).

El rol de la educación en este proceso es considerado fundamental, ya que en ella reside la base para construir escenarios de futuro más sostenibles y equitativos. En este sentido las instituciones de formación de profesorado son agentes clave y por estas razones la UNESCO $(2005,2006)$ recomienda que las naciones incluyan las instituciones de formación de profesorado en sus planes de sostenibilidad nacional.

1. Se define desarrollo sostenible como un proceso de aprendizaje social, que es adaptativo y requiere flexibilidad, creatividad y reflexión crítica. Utilizaremos como sinónimo el termino sostenibilidad. 


\section{¿Por qué hablar de competencias profesionales en Educación para la Sostenibilidad?}

Mientras los sistemas educativos formales tienden a dar prioridad a la adquisición de conocimientos, en detrimento de otras formas de aprendizaje, es necesario concebir la educación como un todo, y es en esta concepción que deben buscar inspiración y orientación las reformas educativas, tanto en la elaboración de los programas como en la definición de las nuevas políticas pedagógicas. El informe Delors (1996) «La educación encierra un tesoro», informe requerido por la UNESCO con la finalidad de orientar la educación del siglo XXI, propone el concepto de educación a lo largo de la vida como la clave para entrar en el siglo XXI, en el que todo puede ser ocasión para aprender y desarrollar las capacidades del individuo. En el informe se destaca la idea que la educación a lo largo de la vida debe basarse en cuatro pilares: aprender a conocer, aprender a hacer, aprender a vivir juntos y aprender a ser. $\mathrm{El}$ aprender a hacer nos remite al concepto competencia, ya que se trata de adquirir no solamente una calificación profesional, sino una competencia que capacite al individuo para hacer frente a un gran número de situaciones, a trabajar en equipo y aprender a hacer en el marco de las diferentes experiencias sociales o de trabajo.

Podemos pues considerar que la mirada competencial en educación prende fuerza a partir del informe Delors y es recogida e institucionalizada por diversos organismos y consejos gubernamentales. En este sentido nos referiremos a la Recomendación del Parlamento Europeo y del Consejo, de 18 de diciembre de 2006, sobre las competencias clave para el aprendizaje permanente (Diario Oficial L 394 de 30.12.2006), en la cual se definen las siguientes competencias clave (CEE, 2006, p. 4):

Una combinación de conocimientos, capacidades y actitudes adecuadas al contexto. Las competencias clave son aquéllas que todas las personas precisan para su realización y desarrollo personales, así como para la ciudadanía activa, la inclusión social y el empleo. El marco de referencia establece ocho competencias clave siguientes: comunicación en la lengua materna; comunicación en lenguas extranjeras; competencia matemática y competencias básicas en ciencia y tecnología; competencia digital; aprender a aprender; competencias sociales y cívicas; sentido de la iniciativa y espíritu de empresa, y conciencia y expresión culturales.

El pensamiento crítico, la creatividad, la reflexión, la iniciativa, la resolución de problemas, la participación, la toma de decisiones y la gestión constructiva de los sentimientos juegan un papel esencial en la educación por competencias y son elementos que harán posible la capacidad para conseguir los objetivos comunes como los procesos democráticos, la productividad económica, la cohesión social, la equidad, los derechos humanos y la sostenibilidad ecológica (CEE, 2006; OCDE, 2002).

Existen numerosas definiciones para el concepto de competencia en educación, la OCDE (2002: 4) en el Informe DeSeCo (Definición y Selección de Competencias) la define como:

Una competencia es más que solamente conocimientos y habilidades. Se trata de la capacidad para satisfacer las demandas complejas, apoyándose en y movilizando recursos psicosociales (incluyendo habilidades y actitudes) en un contexto particular. El desarrollo sostenible y la cohesión social dependen críticamente en las competencias de toda nuestra población -con las competencias entendidas por cubrir conocimiento, habilidades, actitudes y valores.

Mientras se avanza en la definición de las competencias clave en la educación en general, la conceptualización y concreción de la Educación para la Sostenibilidad (ES) también progresa y evoluciona, existiendo un amplio consenso en considerar que la ES se basa en valores de justicia, equidad, tolerancia, suficiencia y responsabilidad (UNESCO, 2004). Promueve la igualdad entre hombres y mujeres, la cohesión social y la reducción de la pobreza, asigna un lugar prioritario al cuidado, la integridad y la honradez. Se sustenta en principios propicios a modos de vida sostenibles, la democracia y al bienestar de los seres humanos (UNESCO, 2009). 
Uno de los hechos más destacables en la ES de este inicio de siglo lo constituye la declaración de la Década de las Naciones Unidas de la Educación para el Desarrollo Sostenible (DEDS, 2005-2014), coordinada por la UNESCO, y que tiene por objeto integrar los principios, valores y prácticas del desarrollo sostenible en todos los aspectos de la educación y el aprendizaje, para abordar los problemas sociales, económicos, culturales y ambientales del siglo XXI.

La visión básica de la DEDS (UNESCO, 2004) es un mundo donde cada persona tiene la oportunidad de beneficiarse de la educación y de aprender valores, comportamientos y estilos de vida requeridos para un futuro sostenible y para una transformación social positiva. Así mismo, se definen los roles clave de la educación, siendo considerada como el agente primario de transformación hacia el desarrollo sostenible, incrementando las capacidades de las personas para transformar sus visiones de la sociedad en realidad.

Realizando un simple análisis comparativo entre los documentos citados y los objetivos de la Década para la Educación para el Desarrollo Sostenible, se puede observar la gran coherencia que existe entre ellos al tener como finalidad la formación de una ciudanía competente para participar de manera activa y responsable en la sociedad, con capacidad de toma de decisiones ante diferentes situaciones y comprometida en la búsqueda de una sociedad más justa, equitativa y sostenible.

\section{El reto de Educar para la Sostenibilidad}

Ante este panorama favorable a la Educación para la Sostenibilidad, nos encontramos con algunos obstáculos y resistencias. Así, en la VI Conferencia Ministerial «Un medio ambiente para Europa» (UNECE, 2007) se identificaron las siguientes preocupaciones con relación al desarrollo de la Educación para la Sostenibilidad en Europa:

- La ES es considerada esencialmente como una educación escolar.

- La carencia de un enfoque institucional global.

- La ausencia de un enfoque sistémico.

- La escasa atención a las instituciones vocacionales y a la formación.

- La ES no forma parte de la formación inicial del profesorado.

- Existe una ausencia de una definición común en relación a las competencias en ES.

Además, diversos informes del Comité Directivo de la UNECE en Educación para el Desarrollo Sostenible (2008a, 2008b, 2009) destacan que:

- La ausencia en la definición de competencias de los educadores es a menudo un obstáculo para la mejora de la calidad de la educación.

- Una de las prioridades para la futura implementación de estrategias para la ES tendría que ser el desarrollo de competencias en ES.

- La mayor parte de los países han tomado medidas para incorporar la ES, pero las cuestiones relacionadas con las competencias adecuadas en materia de ES son todavía un requisito imprescindible.

Por este motivo la UNECE (2011) creó un grupo de expertos en competencias en ES, que recientemente ha publicado un documento sobre las competencias de los educadores en ES, en el que se realizan una serie de recomendaciones a los políticos de todos los países de la Unión Europea para el desarrollo de competencias en todos los sectores de la educación y se identifica una serie de competencias básicas generales en materia de ES para los educadores de todos los niveles y ámbitos educativos. Estas competencias se agrupan en torno a los cuatro pilares del informe Delors (1996), mencionados 
anteriormente, y a tres características esenciales de la ES: un enfoque holístico e integrador, visionar cambio y lograr la transformación.

El grupo de trabajo en Formación Inicial del Profesorado en el IV Congreso Internacional en Educación Ambiental "Tblisiplus30», celebrado en Ahmedabad (India) en 2007, definió las competencias que tendrían que ser consideradas en la formación del profesorado como las siguientes (Hopkins y Ravindranath, 2007):

- La reflexión ética sistemática sobre los valores e intereses relativos a las relaciones humanas y al cuidado de la naturaleza debe ser un componente importante de la competencia del profesorado. Considerar de manera integral la participación activa y la co-construcción de las prácticas educativas y de la ES en la sociedad.

- La capacidad de apoyar y gestionar la diversidad cultural y social.

- La capacidad para identificar las conexiones entre la dimensión sociocultural, la ecológica y la económica del medio ambiente.

- La capacidad para identificar las conexiones entre las condiciones ambientales locales y globales y dibujar inferencias significativas en los contextos de formación.

- La confianza e interés en compartir experiencias, conocimiento, capacidades y valores con los demás.

Existe una problemática a nivel internacional con relación a la definición de las competencias del profesorado en ES y la necesidad de llevar a cabo estudios que puedan contribuir a clarificar este ámbito. La investigación que aquí se presenta emerge de esta necesidad. Aunque existen numerosas investigaciones y documentos sobre los criterios y las competencias para la sostenibilidad pocos son los que hacen referencia a las competencias en ES y se constata que la falta de competencias específicas de los educadores constituye un obstáculo para la mejora de la calidad de los procesos de enseñanza-aprendizaje en ES (UNECE, 2008b). Es necesario reorientar los programas de elaboración de planes de estudio y formación de docentes, con el objetivo de integrar la ES en los programas de formación inicial y de perfeccionamiento para docentes en funciones. Animar a las instituciones de formación de docentes, a los maestros y profesores a crear redes y a elaborar e investigar las prácticas pedagógicas idóneas. En particular, ayudar a los docentes a formular estrategias de ES aplicables a clases numerosas y a evaluar los procesos de aprendizaje de la ES (Declaración de Bonn, UNESCO, 2009).

Las competencias en ES en la formación inicial del profesorado devienen una prioridad a nivel nacional e internacional, siendo necesario definir marcos conceptuales que permitan integrar la ES en los diferentes niveles educativos, así como facilitar la formación para disponer de las herramientas para promover una transformación positiva de la educación y la sociedad desde las aulas de Educación Primaria.

Esta investigación pretende responder a las siguientes preguntas:

¿Qué competencias profesionales en Educación para la Sostenibilidad se deberían desarrollar durante la formación inicial del profesorado?

¿Qué visión sobre las competencias profesionales en Educación para la Sostenibilidad tienen los futuros maestros?

Estas preguntas nos llevan a definir los siguientes objetivos:

- Redefinir un marco de competencias profesionales en Educación para la Sostenibilidad en la formación inicial del profesorado.

- Explorar la visión sobre competencias profesionales en Educación para la Sostenibilidad de los futuros maestros. 


\section{MARCO TEÓRICO DE LA INVESTIGACIÓN}

La redefinición del marco competencial en Educación para la Sostenibilidad en la formación inicial del profesorado se ha realizado tomando como base tres referentes teóricos importantes:

- CSCT Project (Sleurs, 2007)

- Modelo ACES (Junyent, Geli y Arbat, 2003)

- Criterios de Calidad para Escuelas Sostenibles (Breiting, Mayer y Mogensen, 2005)

A continuación mostramos una breve síntesis de estos referentes.

\section{CSCT Project: «Competency based curriculum development for education for sustainable development in initial teaching training and in-service training institutions»}

Cabe destacar que uno de los pocos estudios y documentos que existe a nivel internacional sobre las competencias del profesorado en Educación para la Sostenibilidad es el CSCT Project EU-COM2: Competencies for ESD (Education for Sustainable Development) teachers. A framework to integrate ESD in the curriculum of teacher training institutes (Sleurs, 2007), aunque se contemplan otras investigaciones y documentos, que aunque su objetivo central no son las competencias profesionales nos ofrecen un marco general para la ambientalización curricular y la integración de la sostenibilidad en la educación formal.

El proyecto CSCT se desarrolló como respuesta a la convocatoria de los Ministros de la CEPE/ ONU para el Medio Ambiente en 2003 para incluir la ES en los programas educativos desde el nivel preescolar hasta el superior y la educación de adultos. Se consideró esencial el replanteamiento de la formación docente y la elaboración de un marco curricular de carácter competencial en la formación del profesorado, inicial y permanente. El proyecto financiado por la Comisión Europea entre 2004 y 2007 agrupó 14 instituciones europeas de formación de profesorado, siendo uno de los resultados del proyecto la elaboración de un modelo dinámico para las competencias en Educación para la Sostenibilidad en la formación del profesorado.

En este modelo se consideran dos dimensiones interdependientes:

- Dimensión profesional: El maestro como individuo, como miembro de la institución educativa y como miembro de la sociedad.

- Dimensión competencias generales para la ES: Enseñar (comunicar), reflexionar/visionar y networking (establecer redes y trabajo en equipo).

La interrelación entre las dos dimensiones, junto con la consideración central de la orientación de futuros y la contextualización (local-global-local), a lo largo de los procesos de enseñanza y aprendizaje, llevan a la definición de las competencias específicas del profesorado para la ES. Estas competencias específicas se agrupan en cinco dominios competenciales (Sleurs, 2007: 42-3):

- Conocimiento: conocimiento conceptual, factual y relacionado con la acción, teniendo en cuenta la construcción individual y la estructura social del conocimiento.

- Pensamiento sistémico: la complejidad y la interconectividad de nuestro mundo actual pide pensar en sistemas

- Emociones: pensar, reflexionar, valorar, tomar decisiones y actuar están ligados inseparablemente a las emociones. La competencia emocional es indispensable para el compromiso y el proceso que ha de conducir a la ES. La empatía y la compasión tienen un papel fundamental.

- Ética y valores: las normas, los valores, las actitudes, las creencias y las asunciones guían nuestra percepción, pensamiento y decisiones y acciones. El principio más importante que guía la ES es la equidad (social, intergeneracional, de género, comunitaria...). 
- Acción: es el proceso en que todas las otras competencias de los otros cuatro dominios se combina en creaciones, participación y cooperación significativas. Necesita de unas capacidades, competencias y habilidades prácticas.

El proyecto CSCT pone énfasis en el enfoque interdisciplinar y en los procesos de investigaciónacción y considera aspectos científicos, económicos, sociales, políticos, culturales y éticos del desarrollo sostenible.

\section{Modelo ACES: Ambientalización Curricular de los Estudios Superiores}

El trabajo realizado por la Red ACES con relación a la ambientalización curricular de los Estudios Superiores (Arbat y Geli, 2002; Junyent, Geli y Arbat, 2003; Geli, Junyent y Sánchez, 2003, 2004) constituye un referente a nivel internacional en campo de la ambientalización curricular y representa un documento base para trabajos en el ámbito. La Red ACES definió la ambientalización curricular de los estudios superiores como (Junyent, Geli y Arbat, 2003: 21):

Un proceso continuo de producción cultural que tiende a la formación de profesionales comprometidos con la búsqueda permanente de las mejores relaciones posibles entre la sociedad y la naturaleza, atendiendo a los valores de justicia, solidaridad y equidad, aplicando los principios éticos universalmente reconocidos y el respeto a las diversidades.

Implica la formación de profesionales que puedan vivenciar situaciones reales que propicien la reflexión sobre las dimensiones afectivas, estéticas, éticas de las relaciones interpersonales y con la naturaleza. Esto requiere la realización de trabajos que faciliten el contacto con los problemas socio-ambientales en el propio escenario en que suceden.

En base a esta definición, la caracterización de un estudio ambientalizado viene dada por la integración de diez características:

1. Paradigma de la complejidad: integrar la complejidad como paradigma interpretativo de la realidad y del pensamiento.

2. Flexibilidad y permeabilidad en el orden disciplinar: permitir una apertura a nuevas disciplinas, así como una flexibilidad y permeabilidad disciplinaria a distintas escalas, ya sea transdisciplinaria, interdisciplinaria o pluridisciplinaria.

3. Contextualización: en el espacio (local y global) y en el tiempo (históricamente, en el presente y con visión de futuro).

4. Tener en cuenta al sujeto en la construcción del conocimiento: considerar la persona (individuo o colectivo) como agente activo en la construcción del conocimiento.

5. Considerar los aspectos cognitivos, afectivos y de la acción de las personas, favoreciendo un desarrollo integral.

6. Coherencia y reconstrucción entre teoría y práctica: favorecer la relación entre el discurso y la acción a nivel institucional, docente y de investigación.

7. Orientación prospectiva de escenarios alternativos: favorecer el pensamiento crítico y la toma responsable de decisiones considerando posibles escenarios futuros.

8. Adecuación metodológica: adecuar la metodología propia de la disciplina a la que propone la ES.

9. Generación de espacios de reflexión y participación democrática que conduzcan a la acción para el cambio hacia la sostenibilidad (a nivel institucional y de aula) implicando a todos los colectivos de la comunidad universitaria.

10. Compromiso para la transformación de las relaciones sociedad-naturaleza: favorecer el compromiso hacia una equidad social, un desarrollo económico y un equilibrio ecológico. 


\section{Criterios de Calidad para Escuelas Sostenibles}

La red ENSI (Environment and School Initiatives) inició la red europea School Development through Environmental Education (SEED) para promover la Educación Ambiental como motor para el desarrollo escolar. En el marco de la red de acción COMENIUS de la Comisión Europea, SEED llevó a cabo entre 2002 y 2005 un conjunto de proyectos escolares multilaterales, contribuyendo al mundo de la investigación y a la mejora de la práctica docente. Uno de los resultados de este proceso fue la publicación de los Criterios de Calidad para Escuelas Sostenibles (Breiting, Mayer y Mogensen, 2005). Se tomaron como referentes estos criterios de calidad porque pueden informarnos de las competencias profesionales necesarias para conseguir la implementación de estos criterios. De acuerdo con Breiting et al. (2005: 11), una escuela dedicada a la Educación para la Sostenibilidad se centra en:

La enseñanza para el futuro, con la invitación de los estudiantes y profesores a entrar a una «cultura de la complejidad", mediante la utilización de pensamientos críticos para explorar y retar, con valores aclaradores, los valores prácticos de enseñanza y de participación y revisan todas las asignaturas y la pedagogía a la luz de la Educación para el Desarrollo Sostenible. Estos pueden ser todos los elementos centrales en el desarrollo de la competencia de las acciones estudiantiles.

Estos criterios se consideran puntos de partida para el aprendizaje, la reflexión y discusión en el contexto educativo, promoviendo a la vez la participación crítica, la evaluación y la revisión por parte de los agentes implicados. En el documento se definen 3 grupos de criterios:

- Criterios de calidad relacionados con la calidad de los procesos de enseñanza-aprendizaje: procesos de enseńanza-aprendizaje, perspectivas futuras, complejidad, pensamiento crítico, clarificación y la construcción de valores, procesos de participación y acción, materia de estudio.

- Criterios de calidad relacionados con la política y la organización escolar: administración, planificación y gestión de la escuela, clima escolar, reflexión y evaluación de los proyectos de ES en el centro escolar.

- Criterios de calidad relacionados con las relaciones externas de la escuela: cooperación dentro de la comunidad y trabajo en redes.

La propuesta de SEED-ENSI (Breiting, Mayer y Mogensen, 2005), junto con el modelo ACES (Junyent, Geli y Arbat, 2003) y el modelo CSCT (Sleurs, 2007), han sido utilizados en esta investigación como punto de partida en la redefinición del marco de competencias profesionales en ES.

\section{REDEFINICIÓN DE UN MARCO DE COMPETENCIAS PROFESIONALES EN EDUCACIÓN PARA LA SOSTENIBILIDAD EN LA FORMACIÓN INICIAL DEL PROFESORADO}

A partir del estudio exhaustivo de diferentes definiciones del término competencia y de la triangulación de los diferentes referentes y modelos teóricos citados anteriormente, se propone la siguiente definición para la competencia profesional en Educación para la Sostenibilidad:

La competencia de educar a la ciudadanía para construir un mundo más justo, equitativo y sostenible. Implica la combinación de habilidades prácticas, conocimientos, motivación, valores éticos, actitudes y emociones, y otros componentes sociales y de comportamiento que se movilizan conjuntamente ante un reto de sostenibilidad a nivel social, ambiental y/o económico que se sitúa en un determinado contexto cultural y en el entorno educativo. Son las competencias que tendrían que contribuir a mejorar la calidad de vida de la población y a construir una sociedad y un futuro más sostenible a través de la educación. 
Podemos complementar esta definición indicando que se trata no de una única competencia, sino de una constelación de competencias que deberían contribuir a la mejora de la calidad de vida de la población.

En el ejercicio de concreción de la definición identificamos una serie de competencias profesionales, el conjunto de los cuales dota de entidad a la competencia profesional global en ES. Estas competencias profesionales se conceptualizan como las capacidades que debe desarrollar el/la docente en su ejercicio profesional para incorporar una dimensión de ES en el proceso de enseñanza-aprendizaje en Educación Primaria. El marco competencial que proponemos viene configurado por las siguientes competencias (figura 1):

- Visionar escenarios de futurolalternativos: La capacidad de promover en el alumnado la comprensión de los diferentes futuros posibles y el trabajo con visiones y escenarios, buscando caminos alternativos y cambios para el futuro.

- Contextualizar: La capacidad de trabajar con el alumnado las diferentes dimensiones de un problema o acción, la dimensión espacial (local-global) y la dimensión temporal (pasado, presente y futuro).

- Trabajar y vivir con la complejidad: La capacidad de identificar y conectar las dimensiones ecológica, económica y social de un problema. El profesorado y el alumnado aceptan la incertidumbre y se generan las condiciones para el pensamiento sistémico en el entorno escolar. Se fomenta la capacidad de mirar a las múltiples causas y efectos cuando se exploran y se participa en situaciones.

- Pensar críticamente: Crear las condiciones para el pensamiento crítico en el entorno escolar para cuestionarse asunciones y reconocer y respetar diferentes tendencias en diferentes situaciones. Promover en el alumnado la reflexión sobre las suposiciones que se encuentran debajo de cada crítica, opinión o manera de ver.

- Tomar decisiones, participar y actuar para el cambio: Promover en los alumnos la capacidad de moverse de la conciencia a la acción, compartir responsabilidades e involucrarse en acciones conjuntas. La participación proporciona la motivación para discutir, encontrar soluciones y actuar en un contexto determinado.

- Clarificar valores: El profesorado es capaz de promover la clarificación de valores y comportamientos hacia la sostenibilidad reforzando la reflexión, el respeto mutuo y la comprensión de otros valores entre el alumnado.

- Establecer diálogo entre disciplinas: Desarrollar una enseñanza y aprendizaje basado en un diálogo entre disciplinas, planteado con un enfoque integrador e interdisciplinar.

- Gestionar las emociones: Promover en el alumnado la capacidad de escuchar las propias emociones, preocupaciones, inquietudes y a utilizarlas como medio para llegar a un conocimiento más profundo de los problemas y las situaciones, desarrollando resiliencia.

Este marco competencial puede desarrollarse en cuatro esferas de acción: el/la maestra como individuo, como miembro de la institución educativa, como miembro de la sociedad y como ciudadano/a del mundo, aunque cabe destacar que la esfera en que se sitúa este estudio es el de el/la docente como miembro de la institución educativa. Este marco competencial ha sido validado por expertos en ES del Grupo Còmplex de la Universitat Autònoma de Barcelona. 


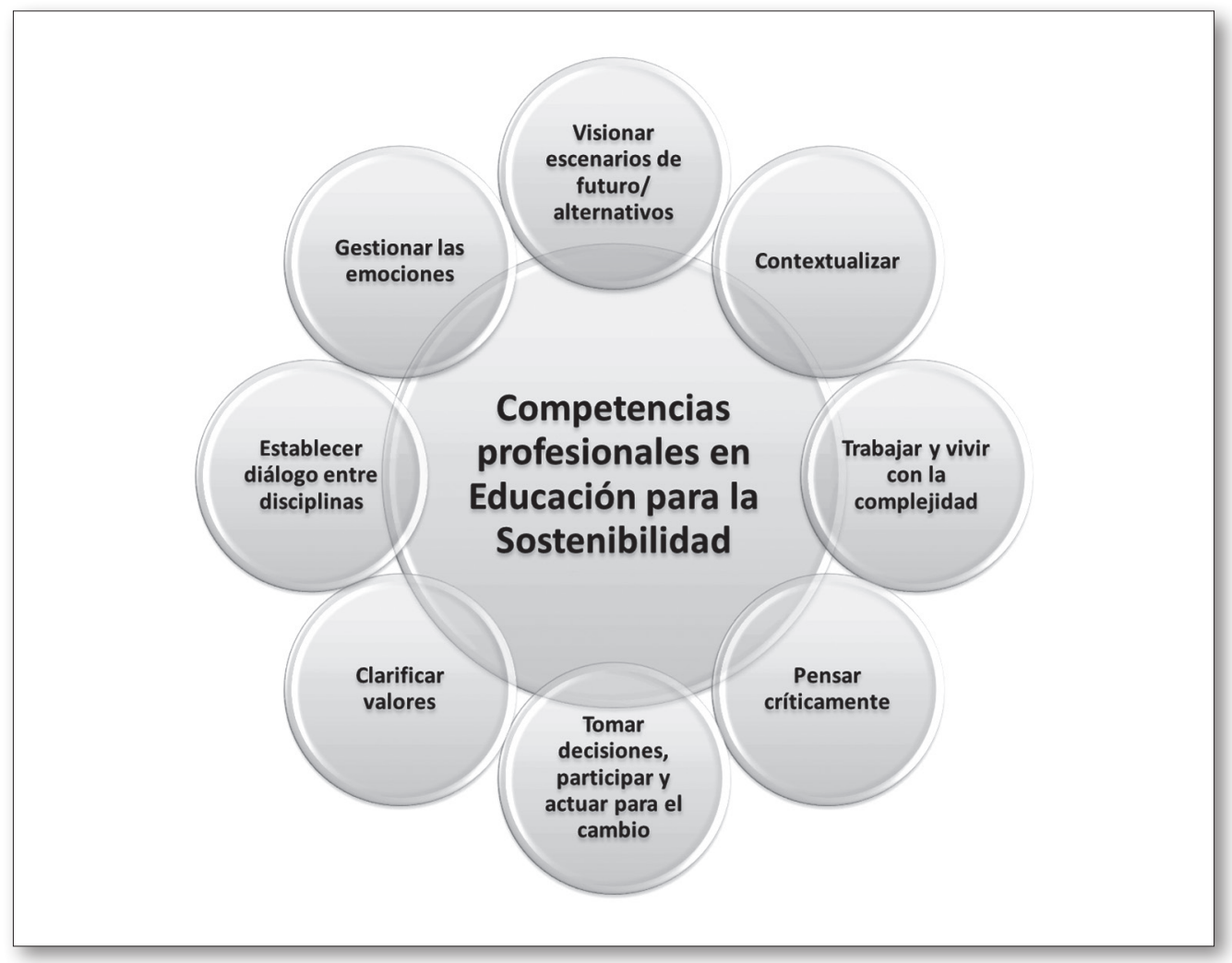

Fig. 1. Competencias profesionales en Educación para la Sostenibilidad

\section{METODOLOGÍA DE LA INVESTIGACIÓN}

Con la intención de explorar la visión sobre las competencias profesionales en ES de los futuros maestros, que corresponde al segundo objetivo que nos planteamos en el trabajo, se ha llevado a cabo una investigación situada en el paradigma interpretativo y concretada en un estudio de caso exploratorio. Desde esta perspectiva, la realidad y el conocimiento son construidos subjetivamente, siendo el principal objetivo de la investigación hacer aflorar y explorar los puntos de vista de aquellos que son interrogados (Mogensen, Mayer, Breiting y Varga, 2007).

El instrumento utilizado ha sido un cuestionario al que han respondido un grupo de 32 alumnos de 3. ${ }^{\circ}$ de Magisterio de la diplomatura de Educación Primaria (curso académico 2009-2010) en el espacio de la asignatura de Didáctica de las Ciencias Experimentales. La selección de este grupo ha sido por conveniencia, y porque, como señalan algunos autores (Gairín, 2009; Sanmartí, 2007), no se puede hablar del desarrollo de competencias profesionales hasta estadios finales de la formación.

Siguiendo el modelo PISA (OCDE, 2006) y otros referentes como Gairín (2009) y Sanmartí (2007), es necesario partir de situaciones reales y contextualizadas para poder valorar el grado de desarrollo competencial. A partir de un material de estímulo como puede ser un texto, un artículo, un gráfico... se establece el contexto de evaluación (OCDE, 2006) y se generan situaciones propias al entorno educativo y situaciones comunes a la vida profesional real. Una vez identificada esta situaciónproblema real y contextualizada, a partir de una serie de preguntas que requieran la movilización de diferentes saberes (OCDE, 2002) nos podemos aproximar a la visión de las futuras docentes sobre las competencias profesionales en ES. Nos referimos a una aproximación, ya que reiteramos que estas solamente pueden ser evaluadas en el contexto de acción educativa. 


\section{El cuestionario}

El cuestionario diseñado consta de un texto inicial dónde se expone una situación para el estudio del medio en una escuela de Educación Primaria y partiendo de este contexto se realizan una serie de preguntas abiertas.

El texto es el siguiente:

\section{Diario de Marta Carreras. Maestra de 5. ${ }^{\circ}$ de primaria de la escuela municipal de Sallent}

Soy Marta, maestra de Educación Primaria desde hace 5 años y ya hace 2 que trabajo en la misma escuela. El año pasado, en la reunión de ciclo nos pidieron a los profesores de la Comisión de Ciencias que preparáramos un nuevo proyecto monográfico (eje temático) para el ańo siguiente.

Justo al lado de la escuela hay un bosque municipal, el Bosquet de l'Aplec, y me pareció una buena oportunidad pensar en algún proyecto relacionado con este espacio. Mi idea interesó a los otros maestros, así que este curso el eje temático que trabajamos lo hemos titulado «¡Hagamos vivir y sonreír al bosquecito!». Este monográfico está relacionado con el currículum actual de Educación Primaria.

En este artículo se presentan los resultados obtenidos a través del análisis de las preguntas 1 y 2 del cuestionario, que son las que pueden aproximarnos a la visión sobre las competencias profesionales en ES de los futuros maestros y maestras:

- Pregunta 1. Imagínate que participas en este proyecto, ¿podrías definir 4 objetivos de aprendizaje que te plantearías alcanzar en su desarrollo?

- Pregunta 2. ¿Cuáles son los contenidos que los/las alumnos/as podrían aprender en un proyecto como este?

El estudio se ha centrado en los objetivos de aprendizaje y los contenidos que los futuros maestros priorizarían o promoverían trabajar con su alumnado en una situación cercana a la realidad profesional. Entendemos que estos objetivos y contenidos nos permiten realizar una aproximación a las diferentes competencias profesionales que activarían los estudiantes en una situación próxima a la práctica educativa. Por lo tanto estas preguntas nos permiten explorar su visión sobre estas competencias profesionales en ES, y por consiguiente responder a la segunda pregunta de investigación.

La situación real planteada corresponde a un proyecto de estudio del medio, en el que no se hace referencia a la ES con el propósito de no influir en las respuestas de los estudiantes, es decir, poder valorar como cuando se les propone desarrollar un proyecto de estudio del medio natural no se limitan solamente a contenidos y objetivos de la disciplina de Ciencias Naturales, sino que incorporan componentes de la ES, sin que se haya hecho explicito ni en la situación ni en el cuestionario.

\section{El análisis de los datos}

El análisis de los datos obtenidos en las preguntas del cuestionario se ha basado en un análisis cualitativo. Se ha utilizado el programa Atlas.ti del tipo CAQDAS (Software para el análisis de datos cualitativos asistidos por ordenador), que contribuye a sistematizar el análisis de datos cualitativos mediante el estudio de múltiples ópticas que aportan una mejor explotación de los mismos para las finalidades de la in- 
vestigación (Chacón, 2004). En este proceso se ha utilizado el manual de Muñoz (2005) para orientar el tratamiento de los datos. Atlas.ti ha permitido codificar las unidades de significado, los fragmentos de texto con significado. Se ha considerado que una unidad de significado es un objetivo de aprendizaje o un contenido propuesto y listado por cada estudiante en las preguntas 1 y 2 del cuestionario.

El análisis de datos ha sido realizado según la conceptualización del término competencia del informe $\mathrm{DeSeCo}$ y posteriormente los resultados se trasladan y relacionan con el marco competencial en ES definido en el estudio.

Las categorías se establecen de manera deductiva a partir de la definición de competencia: «las competencias clave implican una movilización de habilidades cognitivas y prácticas, habilidades creativas y de otros recursos psicosociales como actitudes, motivación y valores» (OCDE, 2002: 8). La siguiente tabla muestra las categorías y una breve descripción (tabla 1):

Tabla 1.

Definición de categorías

\begin{tabular}{|l|l|}
\hline Conocimientos & \multicolumn{1}{|c|}{ Descripción de las categorías } \\
\hline Habilidades prácticas & $\begin{array}{l}\text { Se refiere a los contenidos conceptuales, el hecho de conocer unos deter- } \\
\text { minados conceptos. }\end{array}$ \\
\hline $\begin{array}{l}\text { Se refiere a aquellos contenidos procedimentales como técnicas de trabajo } \\
\text { general, de resolución de problemas, etc. A capacidades, como la capa- } \\
\text { cidad de comunicación, de cambio social, de acción, etc. Son procedi- } \\
\text { mientos, métodos, estrategias, herramientas y técnicas que van más allá } \\
\text { del hecho de saber conocer. }\end{array}$ \\
\hline Valores éticos & $\begin{array}{l}\text { Se refiere a principios normativos que presiden y regulan el comporta- } \\
\text { miento de las personas en cualquier momento y situación. } \\
\text { Los valores hacen referencia a cómo cada uno piensa que deberían ser las } \\
\text { relaciones y las influencias mutuas entre personas, a la sociedad, y entre la } \\
\text { sociedad y la naturaleza. }\end{array}$ \\
\hline Actitudes & $\begin{array}{l}\text { Se refiere a la tendencia a comportarse de manera persistente y consciente } \\
\text { ante estímulos y situaciones concretas, exteriores o interiores. Es una defi- } \\
\text { nición que sitúa las actitudes en un referente más concreto que los valores. } \\
\text { Por ejemplo, la disposición a valorar a los demás, a dialogar, a participar, } \\
\text { constituyen actitudes necesarias para concretar el valor cooperación. }\end{array}$ \\
\hline Emociones & $\begin{array}{l}\text { Hace referencia al conocimiento de uno mismo, a los sentimientos y emo- } \\
\text { ciones que nos mueven en nuestra propia acción. }\end{array}$ \\
\hline
\end{tabular}

Aunque es común denominar valores éticos y actitudes en un mismo grupo, se ha optado por tratarlos de manera separada por considerar que tienen especificidades, tal como se muestra en la descripción (tabla 1), aunque entendemos que en la práctica a menudo se sitúan en la misma categoría por ser contenidos que están relacionados y son interdependientes.

Las subcategorías han sido construidas de manera inductiva a partir de las respuestas de los estudiantes, siguiendo un proceso de comparación constante entre unidades de significado. Estas subcategorías se muestran en la tabla 2 , junto a una ejemplificación de unidades de significado. 
Tabla 2.

Definición de subcategorías

\begin{tabular}{|c|c|c|}
\hline Categoría & Subcategorías & Ejemplificación unidades de significado \\
\hline \multirow{3}{*}{ Conocimientos } & $\begin{array}{l}\text { Conocimientos sobre el medio } \\
\text { natural }\end{array}$ & $\begin{array}{l}\text { E5: «Conocer el entorno cercano a la escuela». } \\
\text { E7: «Conocer algunas especies vegetales del bosque». } \\
\text { E28: «Conocer la flora del entorno». }\end{array}$ \\
\hline & $\begin{array}{l}\text { Conocimientos sobre el medio } \\
\text { ambiente y las problemáticas } \\
\text { ambientales }\end{array}$ & $\begin{array}{l}\text { E6: «Usos del bosque por parte del hombre: lúdico, industriales...». } \\
\text { E17: «Acciones humanas que perjudican al medio ambiente». } \\
\text { E28: «Residuos y contaminación». }\end{array}$ \\
\hline & $\begin{array}{l}\text { Conocimientos que } \\
\text { interrelacionan el medio natural } \\
\text { con otros aspectos }\end{array}$ & $\begin{array}{l}\text { E2: «Conocer los beneficios que aporta el bosque al entorno } \\
\text { urbano». } \\
\text { E8: «Los beneficios y funciones del bosque para el entorno». } \\
\text { E24: «Participar de manera interdisciplinaria con otras áreas». }\end{array}$ \\
\hline \multirow{6}{*}{$\begin{array}{l}\text { Habilidades } \\
\text { prácticas }\end{array}$} & $\begin{array}{l}\text { Identificación y reconocimiento } \\
\text { de la diversidad natural }\end{array}$ & $\begin{array}{l}\text { E13: «Identificar las plantas y seres vivos que hay en el bosque». } \\
\text { E21: «Analizar el funcionamiento de este ecosistema». } \\
\text { E32: «Explorar, identificar y experimentar con la flora y fauna de la } \\
\text { zona». }\end{array}$ \\
\hline & Capacidad de acción & $\begin{array}{l}\text { E10: «Proponer formas de mejorar la salud del bosque». } \\
\text { E12: "La implicación para mejorar el entorno». } \\
\text { E24: «Desarrollar acciones de respeto y cuidado del medio } \\
\text { ambiente». }\end{array}$ \\
\hline & $\begin{array}{l}\text { Reconocimiento de la } \\
\text { interacción hombre-naturaleza } \\
\text { y sus efectos }\end{array}$ & $\begin{array}{l}\text { E2: «Diferenciación entre bosque natural y bosque plantado». } \\
\text { E4: «Analizar cuál es el estado de salud del bosquecito». } \\
\text { E29: «Valorar el impacto humano». }\end{array}$ \\
\hline & Técnicas de trabajo general & $\begin{array}{l}\text { E4: «Seguimiento y conocimiento del método científico». } \\
\text { E15: «La experimentación. La observación». } \\
\text { E26: «Trabajar cooperativamente». }\end{array}$ \\
\hline & $\begin{array}{l}\text { Resolución de problemas } \\
\text { ambientales }\end{array}$ & $\begin{array}{l}\text { E3: «Identificar los posibles problemas ambientales que afectan o } \\
\text { pueden afectar». } \\
\text { E3: «Analizar posibles soluciones para los problemas ambientales». } \\
\text { E28: «Detectar problemas ambientales». }\end{array}$ \\
\hline & Capacidad de comunicación & $\begin{array}{l}\text { E4: «Debate y diálogo. Conversaciones sobre temas relativos a los } \\
\text { bosquecito». }\end{array}$ \\
\hline \multirow{3}{*}{ Valores éticos } & Concienciación ambiental & $\begin{array}{l}\text { E19: «Concienciar de la importancia del mantenimiento de los } \\
\text { bosques». } \\
\text { E25: «Identificarse con el entorno para ser conscientes de que } \\
\text { debemos preservarlo entre todos». } \\
\text { E27: «Concienciar de la importancia de la flora y la fauna del } \\
\text { bosque». }\end{array}$ \\
\hline & $\begin{array}{l}\text { Responsabilidad individual } \\
\text { y colectiva }\end{array}$ & $\begin{array}{l}\text { E1: «Ser conscientes y reconocer las actividades que son positivas o } \\
\text { negativas para el bosque». } \\
\text { E12: «La responsabilidad ante la sociedad». } \\
\text { E18: «Reconocer cuáles acciones son positivas y cuáles negativas } \\
\text { para el medio natural para concienciarse de nuestra responsabilidad } \\
\text { como ciudadanos». }\end{array}$ \\
\hline & $\begin{array}{l}\text { Valor positivo para el ser } \\
\text { humano y la sociedad }\end{array}$ & $\begin{array}{l}\text { E2: «Identificación/reconocimiento de las aportaciones beneficiosas } \\
\text { del paraje a la salud de los habitantes del entorno». } \\
\text { E6: «Conocer el entorno natural cercano para apreciar su valor en } \\
\text { la vida de las personas». } \\
\text { E6: «Beneficios y valores: el bosque como entorno necesario para } \\
\text { nuestra vida». }\end{array}$ \\
\hline
\end{tabular}




\begin{tabular}{|c|c|c|}
\hline Categoría & Subcategorías & Ejemplificación unidades de significado \\
\hline \multirow{5}{*}{ Actitudes } & Respeto hacia el medio & $\begin{array}{l}\text { E14: «Respetar la naturaleza y el patrimonio local». } \\
\text { E16: «Aprender a valorar el entorno y respetarlo». } \\
\text { E26: «Propiciar una buena actitud de respeto a los bosques». }\end{array}$ \\
\hline & $\begin{array}{l}\text { Compromiso, implicación y } \\
\text { participación activa }\end{array}$ & $\begin{array}{l}\text { E2: «Propuesta de acciones de preservación de un entorno natural e } \\
\text { implicación personal en éstas». } \\
\text { E12: «La implicación para mejorar el entorno». } \\
\text { E13: «Compromiso e implicación en el cuidado del bosque». }\end{array}$ \\
\hline & El cuidado del entorno & $\begin{array}{l}\text { E3: «Reducción de los residuos del bosque, limpieza del entorno...». } \\
\text { E19: «Eso que podemos hacer para tener un buen cuidado del } \\
\text { bosque». } \\
\text { E22: «Concienciación y cuidado del entorno». }\end{array}$ \\
\hline & $\begin{array}{l}\text { Actitud crítica y de mejora del } \\
\text { mundo }\end{array}$ & $\begin{array}{l}\text { E2: «Capacidad crítica para pensar, reflexionar y actuar». } \\
\text { E7: «Conocimiento del impacto humano sobre el medio para } \\
\text { favorecer una actitud crítica y de mejora del mundo». } \\
\text { E15: «Actitud crítica». }\end{array}$ \\
\hline & $\begin{array}{l}\text { Convivencia y compartición de } \\
\text { experiencias con los/las demás }\end{array}$ & $\begin{array}{l}\text { E5: «Convivir con los compañeros en un espacio diferente que el } \\
\text { aula». } \\
\text { E16: "Aprender a compartir experiencias y conocimientos». } \\
\text { E16: «Convivencia». }\end{array}$ \\
\hline Emociones & $\begin{array}{l}\text { Sentimiento de pertenencia al } \\
\text { entorno }\end{array}$ & $\begin{array}{l}\text { E8: «Aprender a querer el bosque y su utilidad en nuestro } \\
\text { contexto». }\end{array}$ \\
\hline
\end{tabular}

\section{RESULTADOS Y DISCUSIÓN}

Los resultados obtenidos a través del análisis de datos se presentan gráficamente con una red Atlas. ti. La red Atlas.ti ha sido reelaborada para obtener una mejor visualización. Las categorías y subcategorías se organizan de izquierda a derecha y de arriba a abajo por orden de mayor a menor grado de importancia o aparición, con el objetivo de facilitar la visualización de los resultados. Los números que aparecen al lado de cada subcategoría hacen referencia al número de veces que ha sido nombrada dicha subcategoría. 


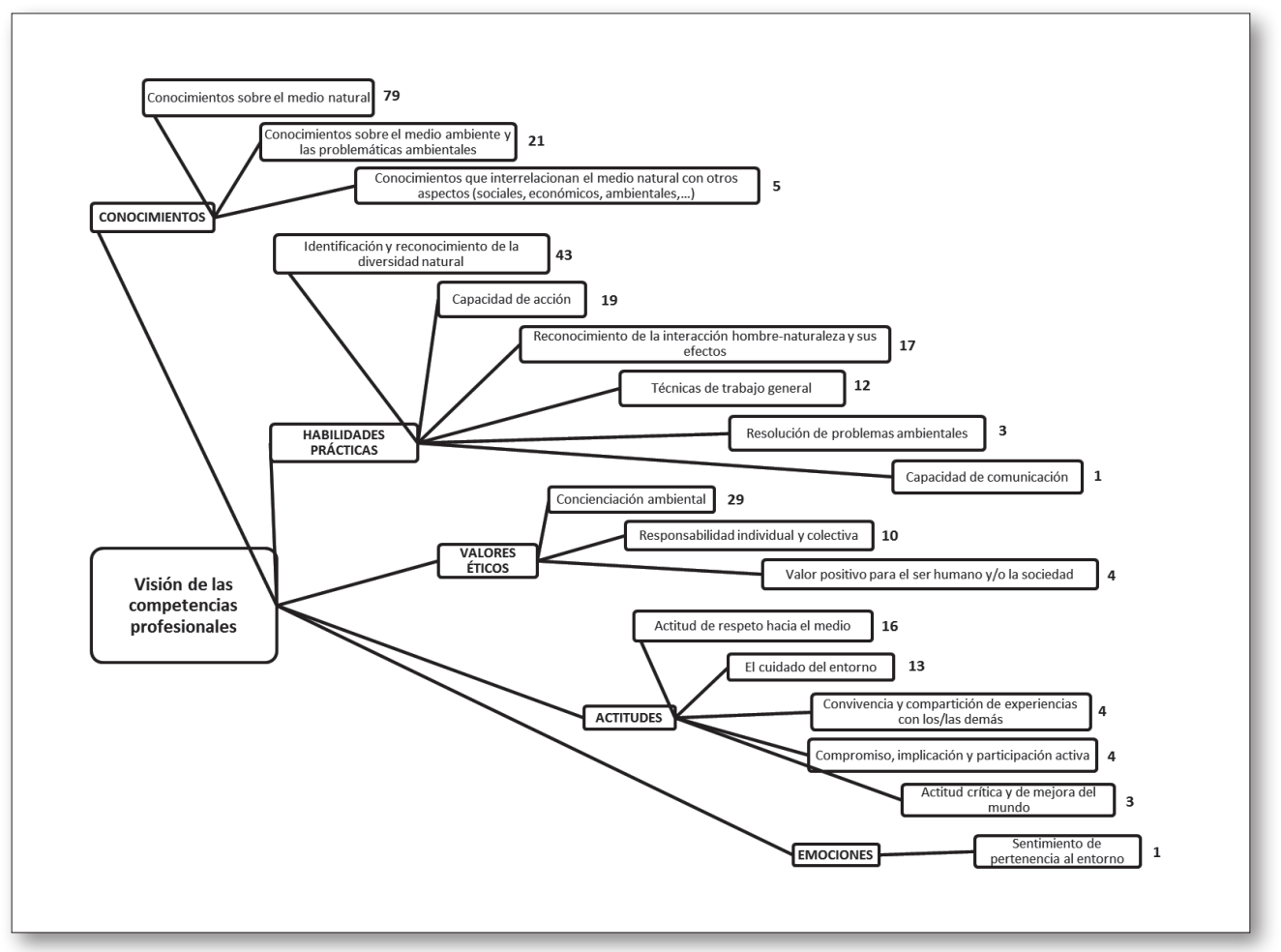

Fig. 2. Red Atlas.ti sobre la visión de las competencias profesionales del proyecto

La figura 2 muestra la visión global de las competencias profesionales para los 32 estudiantes que respondieron al cuestionario.

La adquisición de conocimientos (105) y habilidades prácticas (95) son los objetivos didácticos y los contenidos a los que los futuros maestros darían más importancia alcanzar en un proyecto de estudio del medio como el propuesto. Los valores éticos (43) y las actitudes (40) quedarían en un segundo plano, mientras que a la gestión de las emociones solo se le hace referencia una vez.

Si nos fijamos en los conocimientos cabe destacar el gran peso que dan los futuros maestros a los contenidos conceptuales relacionados con el medio natural, la diversidad natural, la flora y la fauna (79), solo algunos (21) darían importancia a conocimientos sobre el medio ambiente y las problemáticas ambientales como la contaminación de un espacio natural, el impacto ambiental que genera el ser humano, los usos que hace de la naturaleza, etc. Un pequeño número de estudiantes (5) daría importancia a trabajar aspectos de carácter interdisciplinario, trabajando aspectos ambientales pero a la vez aspectos sociales y económicos relacionados con un espacio natural local.

En referencia a las habilidades prácticas, se mantiene como aspecto principal la adquisición de habilidades relacionadas con la naturaleza o la diversidad natural, como la observación, identificación, reconocimiento y análisis de las características y peculiaridades de un espacio natural (43). En segundo lugar, la capacidad de acción (19), proponer actividades y actuaciones de mejora, saber qué hay que hacer para mejorar el estado del espacio, llevar a cabo acciones de mantenimiento del entorno, son algunos de los contenidos que promoverían los futuros maestros en su alumnado. Reconocer la interacción entre el ser humano y la naturaleza así como sus efectos (17), valorar el impacto que se genera, así como las alteraciones que se pueden producir, junto con técnicas de trabajo general (12), como la experimentación, la interpretación de fenómenos, el trabajo en equipo y el método científico son consideradas habilidades importantes. Cabe destacar que un bajo número de estudiantes daría importancia a la resolución de problemas ambientales (3) y capacidades comunicativas (1), al debate y diálogo. 
El valor ético más valorado es la concienciación ambiental (29). La responsabilidad individual y colectiva (10) es otro valor que promover y en menor importancia se consideran también valores éticos relacionados con la valoración de un espacio natural como positivo para el ser humano y la sociedad en general (4).

En referencia a las actitudes, las actitudes positivas de respeto hacia el medio (16), la acción hacia la sostenibilidad y en la preservación y cuidado del entorno (13) son las actitudes que promoverían los futuros maestros en sus alumnos en un proyecto de estudio del medio. Un menor número de estudiantes tiene en cuenta actitudes como las de compartir y convivir con los demás (4), así como la implicación y participación activa en la sociedad (4) y el fomento de actitudes críticas y de mejora del mundo (3).

Las emociones es el elemento competencial menos valorado por los futuros maestros, solo se hace una referencia al hecho de sentirse parte del entorno (1). Por lo tanto, el trabajo de las emociones en el contexto escolar, aprender a gestionarlas y trabajarlas con los compañeros parece ser un aspecto prácticamente no contemplado por el profesorado en formación estudiado.

Se constata que los/las futuros/as docentes otorgan importancia al hecho de promover en su alumnado contenidos conceptuales y procedimentales en detrimento de otros contenidos como los actitudinales, la adquisición de valores o el trabajo de las emociones.

Con el objetivo de visualizar cómo las competencias profesionales en ES definidas en el estudio han sido consideradas por los estudiantes se trasladan las subcategorías obtenidas en el análisis (tabla 2) al marco de competencias profesionales en ES (figura 1).

La correspondencia entre ambas se ilustra en la siguiente tabla (tabla 3):

Tabla 3.

Relación entre las subcategorías y las competencias profesionales en ES

\begin{tabular}{|c|c|}
\hline COMPETENCIAS PROFESIONALES EN ES & SUBCATEGORÍAS ANÁLISIS DE DATOS \\
\hline Clarificar valores & $\begin{array}{l}\text { 1. Concienciación ambiental } \\
\text { 2. Responsabilidad individual y colectiva } \\
\text { 3. Valor positivo para el ser humano y/o la sociedad } \\
\text { 4. Compromiso, implicación y participación activa } \\
\text { 5. Respeto hacia el medio } \\
\text { 6. El cuidado del entorno }\end{array}$ \\
\hline Trabajar y vivir con la complejidad & $\begin{array}{l}\text { 1. Reconocimiento de la interacción hombre-naturaleza y sus efectos } \\
\text { 2. Conocimientos que interrelacionan el medio natural con otros as- } \\
\text { pectos (sociales, económicos, ambientales...) } \\
\text { 3. Conocimientos sobre el medio ambiente y las problemáticas am- } \\
\text { bientales }\end{array}$ \\
\hline Tomar decisiones, participar y actuar para el cambio & $\begin{array}{l}\text { 1. Capacidad de acción } \\
\text { 2. Resolución de problemas ambientales } \\
\text { 3. Compromiso, implicación y participación activa }\end{array}$ \\
\hline Contextualizar & $\begin{array}{l}\text { 1. Reconocimiento la interacción hombre-naturaleza y sus efectos } \\
\text { 2. Conocimientos sobre el medio ambiente y las problemáticas am- } \\
\text { bientales }\end{array}$ \\
\hline Pensar críticamente & Actitud crítica y de mejora del mundo \\
\hline Establecer diálogo entre disciplinas & $\begin{array}{l}\text { 1. Conocimientos que interrelacionan el medio natural con otros as- } \\
\text { pectos (sociales, económicos, ambientales...) }\end{array}$ \\
\hline Gestionar las emociones & Sentimiento de pertenencia al entorno \\
\hline Visionar escenarios de futuro/alternativos & \\
\hline
\end{tabular}


A partir de las relaciones establecidas, en referencia a las competencias profesionales consideradas clave en ES, los estudiantes de educación primaria enfatizan la clarificación de valores, la capacidad de tomar decisiones, participar y actuar para el cambio, así como aspectos relacionados con la complejidad de las situaciones y los problemas ambientales (tabla 3). Por el contrario, la capacidad de contextualizar un problema, visionar escenarios de futuro, el pensamiento crítico, el diálogo entre disciplinas y la gestión de las emociones y preocupaciones son competencias profesionales que prácticamente no son destacadas por los futuros maestros.

Como se ha dicho anteriormente, en la situación de estudio del medio planteada no se hace explícita de manera intencionada la sostenibilidad o la Educación para la Sostenibilidad, con el objetivo de valorar si los estudiantes integran en un proyecto educativo elementos de ES. El análisis nos indica que esta integración no se realiza de manera clara, por lo que podríamos inferir que en la formación inicial del profesorado no se está fomentando una ambientalización curricular que proporcione una visión holística del medio y de su tratamiento educativo.

\section{CONCLUSIONES}

Con relación al primer objetivo de la investigación, redefinir un marco de competencias profesionales en ES en la formación inicial del profesorado, se propone una definición de competencia profesional global en ES y el conjunto de competencias que la forman. Este marco competencial a su vez orienta la exploración de la visión sobre competencias profesionales en ES de los futuros maestros, que constituye el segundo objetivo de esta investigación.

En referencia a este segundo objetivo cabe destacar que los futuros maestros y maestras que han participado en el estudio priorizarían la adquisición de conocimientos y habilidades prácticas en sus alumnos en detrimento de otros tipos de aprendizajes como el trabajo de valores éticos, actitudes favorables y la gestión de las emociones. Los aspectos relacionados con el medio natural y la interacción del ser humano con este son los que consideran más relevantes, juntamente con la capacidad de acción, la concienciación ambiental, la responsabilidad individual y colectiva y actitudes de respeto y cuidado del medio.

Las competencias relacionadas con la interacción social y ciudadana, la interacción entre las personas y los elementos de carácter transversal e interdisciplinar, como el pensamiento crítico y el trabajo en equipo, prácticamente no se tienen en cuenta como a elementos a promover en el alumnado de educación primaria.

Por lo que se refiere a la Educación para la Sostenibilidad y a las competencias profesionales clave en este ámbito, se observa cómo los estudiantes tienden a remarcar aspectos como la sensibilización, la capacidad de acción, la responsabilidad o el cuidado del entorno, dejando de lado otros aspectos como la capacidad de visionar diferentes escenarios de futuro, la interdisciplinariedad, la gestión de las emociones o el fomento del pensamiento crítico, cuando todos estos componentes son esenciales para una integración de la ES.

Algunas de las limitaciones del estudio pueden encontrarse en la situación planteada en el texto del cuestionario, ya que las respuestas de los estudiantes están muy centradas en el ámbito de medio natural. Si el proyecto propuesto fuera una situación de problemática ambiental o sostenibilidad (gestión de residuos, contaminación del medio natural/local, etc.), quedaría por analizar y valorar si las respuestas serían más cercanas o no a competencias en ES.

Por otra parte, en este estudio nos referimos a una aproximación porque se considera que para valorar las competencias profesionales estas deben situarse en un contexto educativo real. Así pues, este análisis podría realizarse en los periodos de prácticas de los estudiantes o en formación permanente de profesorado. 
Los futuros maestros y maestras, durante su formación inicial, tendrían que poder adquirir las competencias profesionales que les capaciten para hacer frente a los diferentes retos de sostenibilidad que se les planteará durante su práctica profesional, y por lo tanto, es necesario poder valorar si esta formación les aporta los conocimientos, las habilidades prácticas, las herramientas, las actitudes y los valores éticos que harán posible que sean capaces de afrontar estos retos. Procesos de ambientalización curricular con un enfoque competencial deberían ser planteados e implementados en las instituciones de formación del profesorado desde y en el contexto particular de cada institución.

Este estudio pretende contribuir a la necesidad de llevar a cabo investigaciones que clarifiquen la definición de competencias del profesorado en ES y que puedan orientar trabajos posteriores sobre cuáles deben ser las estrategias educativas y los instrumentos evaluativos para una integración efectiva de la ES en la formación del profesorado.

Todo ello en el nuevo Espacio Europeo de Educación Superior (EEES) el cual supone una oportunidad para incorporar la sostenibilidad de forma integral en la educación superior. El nuevo modelo en educación superior basado en la enseñanza-aprendizaje por competencias propone algunos cambios importantes e interesantes. Deja atrás un modelo centrado en el profesorado y en la enseñanza, para adoptar un modelo centrado en el alumnado y en su aprendizaje, que promueve de manera activa el pensamiento crítico, la contextualización, la acción, la cohesión social, el que supone una oportunidad y un reto, para introducir en la enseñanza superior lo que la Educación para la Sostenibilidad viene reclamando. El EEES ofrece el marco para que desde las instituciones de educación superior se contribuya de manera activa a formar profesionales en el campo de la educación y ciudadanos en general capacitados para la acción hacia la sostenibilidad.

\section{BIBLIOGRAFÍA}

Bolonia (1999). The Bologna Declaration of 19 June 1999. Disponible en: http://eu.daad.de/imperia/ $\mathrm{md} /$ content/eu/bologna/bolognadeclaration.pdf. (Último acceso, el 20 de noviembre de 2009).

Arbat, E. y Geli, A.M. (eds.) (2002). Aspectos ambientales de las universidades. Girona: Universitat de Girona-Red ACES.

Breiting, S., Mayer, M. y Mogensen, F. (2005). Quality Criteria for ESD-Schools. Vienna: Austrian Federal Ministry of Education, Science and Culture-ENSI.

Cee (2006). Recommendation of the European Parliament and of the Council, of 18 December 2006, on Key Competences for Lifelong Learning. [Official Journal L 394/11 of 30.12.2006]. Disponible en: http://europa.eu/legislation_summaries/education_training_youth/lifelong_learning/c11090_ en.htm. (Último acceso, el 20 de octubre de 2009).

Chacón, E. (2004). El uso de ATLAS/TI como herramienta para el análisis de datos cualitativos en Investigaciones Educativas. I Jornadas Universitarias «JUTEDU2004»: Competencias socio-profesionales de las titulaciones de educación. Madrid: UNED-Madrid.

Delors, J. (1996). L'éducation: Un trésor est caché dedans. Rapport à l'UNESCO de la Commission internationale sur l'éducation pour le vingt et unième siècle. Paris: UNESCO.

GaIrIn, J. (coord.) (2009). Guia per a l'avaluació de competències en l'àrea de ciències socials. Barcelona: Agència per a la Qualitat del Sistema Universitari de Catalunya.

Geli, A.M., Junyent, M. y Sánchez, S. (eds.) (2003). Diagnóstico de la Ambientalización Curricular de los Estudios Superiores. Girona: Universitat de Girona-Red ACES.

Geli, A.M., Junyent, M. y Sánchez, S. (eds.) (2004). Acciones de Intervención y Balance Final del Proyecto de Ambientalización Curricular de los Estudios Superiores. Girona: Universitat de GironaRed ACES. 
Hopkins, C. y Ravindranath, M.J. (2007). Teacher Education - a crucial contribution to DESD. Recommendations. International Conference on DESD, CEE (Ahmedabad, November 2007).

Huckle, J. y Sterling, S. (eds.) (1996). Education for Sustainability. London: Earthscan Publications Limited.

Junyent, M., Geli, A.M. y Arbat, E. (2003). Características de la Ambientalización Curricular: Modelo ACES, en M. Junyent, A.M. Geli y E. Arbat (eds.). Proceso de caracterización de la Ambientalización Curricular de los Estudios Universitarios. Girona: Universitat de Girona-Red ACES.

Mogensen, F., Mayer, M., Breiting, S. y VARGA, A. (2007). Educació per al desenvolupament sostenible: tendències, divergències $i$ criteris de qualitat. Barcelona: Editorial Graó.

Muñoz, J.M. (2005). Manual Análisis cualitativo de datos textuales con ATLAS.ti 5. Bellaterra: Universitat Autònoma de Barcelona.

OCDE (2006). PISA 2006. Marco de evaluación. Conocimientos y habilidades en Ciencias, Matemáticas y Lectura. Disponible en: www.oecd.org/dataoecd/59/2/39732471.pdf. (Último acceso el 20 de enero de 2010).

OCDE (2002). The Definition and Selection of Key Competencies (DeSeCo). Disponible en: www.oecd. org/dataoecd/47/61/35070367.pdf. (Último acceso, el 26 de enero de 2010).

SANMARTí, N. (2007). Evaluar para aprender. 10 Ideas clave. Barcelona: Editorial Graó.

Sleurs, W. (ed.) (2007). Competencies for ESD (Education for Sustainable Development) teachers. A framework to integrate ESD in the curriculum of teacher training institutes. Comenius 2.1 Project 118277-CP-1-2004-BE-. Disponible en: http://www.unece.org/env/esd/inf.meeting.docs/ EGonInd/8mtg/CSCT\%20Handbook_Extract.pdf. (Último acceso, el 15 de octubre de 2009).

Tilbury, D. y Wortman, D. (2004). Engaging People in Sustainability. Commission on Education and Communication. Gland: IUCN.

UNECE (2007). Sixth Ministerial Conference «Environment for Europe». Declaration «Building Bridges to the Future" by Ministers of the region of the United Nations Economic Comission for Europe (UNECE). ECE/BELGRADE.CONF/2007/8. Disponible en: http://www.unece.org/env/efe/ efereform/documentsreformefe.html. (Último acceso el 25 de marzo de 2009).

UNECE (2008a). Comment est enseigné le développement durable? Débat d'experts sur les compétences en matière d'éducation en vue du développement durable dans le secteur de l'éducation. ECE/CEP/ AC.13/2008/7. Disponible en: http://www.unece.org/env/esd/inf.meeting.docs/SC/SC-3/Interventions/SwitzerlandSC3panel.pdf. (Último acceso, el 28 de noviembre de 2009).

UNECE (2008b). Report of the UNECE steering committee on education for sustainable development on its third meeting. ECE/CEP/AC.13/2008/2. Disponible en: http://www.unece.org/?id=14883. (Último acceso, el 25 de noviembre de 2009).

UNECE (2009). Report of the UNECE steering committee on education for sustainable development on its fourth meeting. ECE/CEP/AC.13/2009/2. Disponible desde Internet en: http://www.unece.org/ env/esd/sc.meet2009_4.html. (Último acceso, el 16 de enero de 2010].

UNECE (2011). Learning for the future: Competences in Education for Sustainable Development. ECE/ CEP/AC.13/2011/6. Disponible en: http://www.unece.org/index.php?id=14971. (Último acceso, el 20 de mayo de 2011).

UNESCO (2004). United Nations Decade on Education for a Sustainable Development (2005-2014). Draft Implementation Scheme. Disponible en: http://portal.unesco.org/education/en/file_download.php/e13265d9b948898339314b001d91fd01draftFinal+IIS.pdf. (Último acceso, el 3 de octubre de 2009).

UNESCO (2005). Guidelines and Recommendations for Reorienting Teacher Education to Address Sustainability. Education for Sustainable Development in Action, Technical Paper N. 22, UNESCO Education Sector. Paris: UNESCO. 
UNESCO (2006). Decenio de las Naciones Unidas de la Educación con miras al Desarrollo Sostenible (2004-2015): Plan de aplicación internacional. Sector de Educación de la UNESCO. Paris: UNESCO.

UNESCO (2009). Declaración de Bonn. UNESCO World Conference on Education for Sustainable Development. Disponible en: http:/www.esd-world-conference-2009.org/fileadmin/download/ ESD2009_BonnDeclaration080409.pdf. (Último acceso el 3 de octubre de 2009). 


\section{Professional competencies in Education for Sustainability: an exploratory study of student teachers' views}

\author{
Gisela Cebrián \\ University of Southampton \\ G.Cebrian-Bernat@soton.ac.uk
}

\author{
Mercè Junyent Pubill \\ Departament de Didàctica de la Matemàtica i de les Ciències Experimentals, UAB \\ merce.junyent@uab.cat
}

Education is considered to play a key role in fostering a more sustainable, equitable and socially just society (UNESCO, 2005). Education for Sustainability (EfS) can be seen as an education for a sustainable social transformation, which stimulates critical reflection and the clarification and reassessment of values, and should be innovative and constructive, culturally appropriate and action-orientated (Huckle and Sterling, 1996; Tilbury and Wortman, 2004). Higher Education and especially teacher education institutions are principal agents for a positive transformation towards sustainability, because of its key mission of knowledge generation and transfer through research and teaching (UNESCO, 2005).

However, a number of challenges to foster EfS have been identified (UNECE, 2007), such as EfS not being part of teacher training and a lack of a shared definition on the competencies in EfS. This can represent an obstacle for improving the quality of teaching and learning processes in EfS (UNECE, 2008b). The research presented in this paper emerged from the need to develop a comprehensive framework on the professional competencies in EfS for teachers. This paper is aimed to explore the following research areas: (i) the competencies in EfS that need to be developed during teacher training; and (ii) the views of student teachers on the professional competencies in EfS. This research was exploratory and was placed in the interpretive paradigm.

First, we redefined a conceptual framework on professional EfS competencies for teachers. The development of this framework was conducted through the triangulation of three theoretical frameworks: CSCT Project (Sleurs, 2007); ACES model (Junyent, Geli and Arbat, 2003); and Criteria for ESD-Schools (Breiting, Mayer and Mogensen, 2005). The professional competence in EfS is defined as the competence of educating citizens to build a more just, equitable and sustainable world. It involves the combination of practical skills, knowledge, motivation, ethical values, attitudes, emotions and other social and behavioural components that are mobilised together in a challenge of sustainability at a social, environmental and economic level in a particular cultural context and educational environment. These professional competencies are conceptualised according to the teacher's capacity to promote a type of learning amongst students: visioning of future scenarios; contextualising; working and living with complexity: critical thinking; decision-making, participation and action for change; values clarification; dialogue between disciplines; management of emotions. In turn, the professional competencies' framework guided the exploration of the student teachers' views, which corresponds to the second aim of this research.

Second, in order to explore the views of student teachers on professional competencies in EfS a questionnaire was designed. A group of 32 third-year students on the Bachelor of Primary Education replied to our questionnaire, which consisted of an initial text that presented a situation related to environmental sustainability in a school environment. In this context, a primary school teacher aimed to develop a curricular project based around a natural area close to the school. Trainee teachers were asked questions about their future teaching in this context. This article presents the findings obtained through the analysis of the questionnaires. A qualitative data analysis process allowed us to identify the views of student teachers on the professional competencies in EfS. The findings obtained show that student teachers emphasise the acquisition of knowledge and practical skills in detriment of working on ethical values, attitudes and management of emotions with students. In terms of professional competencies in EfS, student teachers highlight values clarification, decision-making, participation and action for change. However, visioning of future scenarios, critical thinking, interdisciplinary dialogue and the management of emotions are hardly considered. Knowledge acquisition and practical skills are highlighted mainly in reference to the natural world, whereas environmental and interdisciplinary issues are less stressed by student teachers. Problem-solving, communication skills, debate and dialogue are not seen as relevant. In reference to values and attitudes, student teachers emphasise the importance of environmental awareness, positive attitudes, individual and collective responsibility, action towards sustainability, care and respect for the environment. Nevertheless, emotional aspects such as the importance of belonging to the environment are only mentioned once. This research aims to contribute to the definition of professional competencies in EfS. The findings of this research can guide future work on the design and development of educational strategies and assessment tools for the effective integration of EfS in teacher training. 